\title{
Más allá de tópico y foco: Construcciones quiásticas y el morfema $L A$ en el zapoteco del Istmo
}

\section{Introducción}

En este trabajo, ${ }^{1}$ presento un estudio de la estructura informativa en el zapoteco del Istmo (otomangue; ISO 639: ZAI), un idioma zapoteco de la región de Juchitán, Oaxaca, México y que cuenta con aproximadamente 50.000 hablantes. El estudio está basado en un corpus audiovisual recolectado durante 17 meses de trabajo de campo en Juchitán.

Se ha realizado una cantidad considerable de trabajo sobre la documentación y la descripción de las gramáticas de las lenguas zapotecas (por ejemplo, Beam de Azcona 2004, Sonnenschein 2005). Asimismo, varios estudios sobre lenguas zapotecas se han enfocado en el análisis de nociones básicas de la estructura informativa, como tópico y foco (Ward 1987, Lee 1997, 2000, Broadwell 1999a, 1999b, 2002, Lillehaugen 2016). Sin embargo, pocos estudios se han dedicado a analizar la forma en que estos idiomas son utilizados por los hablantes en su vida cotidiana (cf. Sicoli 2007). Además, la investigación tipológica en el área de la estructura informativa sigue siendo difícil debido a la falta de datos discursivos de lenguas tipológicamente diversas. Este trabajo busca ampliar nuestra comprensión de los patrones empíricos de la estructura informativa mediante la documentación y el análisis del ZAI.

A continuación, se presenta un análisis de las relaciones de tópico y foco en contextos narrativos y conversacionales para detenerse en dos fenómenos en particular. En primer lugar, se documenta y se revisa el uso conversacional de una construcción de foco de predicado seguida, dentro del mismo turno de habla, por una construcción de foco estrecho. Se argumenta que este uso sucesivo de dos estructuras de foco distintas funciona como una estructura quiástica por medio de la cual el hablante une dos oraciones y las comunica como una instrucción al interlocutor para que sean interpretadas juntas.

1 El presente capítulo surge de mi tesis doctoral, publicada como Bueno Holle (2019).

Juan José Bueno Holle, Independent Researcher, Sacramento, CA, USA

Ә Open Access. () 2019 Juan José Bueno Holle, published by De Gruyter. (cc) BY-NC-ND This work is licensed under a Creative Commons Attribution-NonCommercial-NoDerivatives 4.0 International License.

https://doi.org/10.1515/9783110605679-005 
En segundo lugar, se analiza una partícula discursiva empleada para marcar distintos tipos de información topical. Se argumenta que esta partícula, la partícula LA, funciona para organizar la conversación y la interacción.

La Sección 2 revisa la estructura focal del ZAI. La Sección 3 aborda el uso coordinado de las estructuras de foco de predicado y de foco estrecho en la conversación. La Sección 4 hace un análisis de los contextos de uso de la partícula discursiva LA. El resumen y las conclusiones se presentan en la Sección 5.

\section{La estructura focal del ZAI}

En esta sección ${ }^{2}$ se analiza la organización de la estructura focal del ZAI con énfasis sobre la manera en que la fonología, la morfología, y la sintaxis interactúan entre sí. Como se verá, los datos del ZAI apoyan la hipótesis de que los hablantes marcan las relaciones de foco principalmente a través de la manipulación del orden de constituyentes y/o mediante la marcación morfológica (para otras lenguas zapotecas, véase Broadwell 1999a, 1999b, 2002, Lee 1997, 2000), más que a través de la prosodia. En el corpus no hay evidencia de la asociación de acentos específicos con las expresiones en foco (ni con los tópicos), aunque los elementos pueden mostrar varias propiedades prosódicas, como la duración, el registro del tono y el rango de tono, que pueden estar relacionadas con la posición dentro de una unidad entonacional dada.

En el análisis y la discusión sobre la estructura focal en ZAI, se seguirá el marco propuesto por Lambrecht (1994). El término estructura focal ("focal structure") se refiere a los medios gramaticales por los cuales una lengua indica lo que se afirma en un enunciado (foco) y lo diferencia de lo que se presupone (tópico). Desde esta perspectiva, los términos tópico y foco no se refieren estrictamente a información nueva o vieja, sino a las relaciones pragmáticas entre los elementos (ya sean nuevos o viejos) del discurso.

El contraste principal en la estructura focal es entre el foco amplio y el foco estrecho. Mientras que para el foco amplio el dominio de foco (focus domain) se extiende sobre más de un constituyente, para el foco estrecho, el dominio de foco se extiende sobre un constituyente solamente. En construcciones de foco amplio, que en ZAI invariablemente son estructuras de verbo inicial, el verbo forma parte de la afirmación. En cambio, en construcciones de foco estrecho, el verbo puede o no formar parte de la afirmación.

2 Esta sección está adaptada y traducida del capítulo 5 de Bueno Holle (2019: 87-103). 
Como se verá en más detalle a continuación, la generalización pertinente entonces es la siguiente: el verbo formará parte del dominio de foco a menos que la construcción sea una construcción con foco estrecho sobre algún constituyente no verbal, en cuyo caso formará parte de la presuposición. A partir de esto, se verá la tipología de la estructura de foco propuesta por Van Valin (1999) para determinar el lugar de ZAI dentro de esa tipología.

\subsection{Foco de predicado}

El foco de predicado se denomina tradicionalmente como construcción de tópico-comentario, donde el sujeto es el tópico y el predicado es un comentario sobre ese tópico. Este es el tipo de foco no marcado. Los siguientes ejemplos de Lambrecht (1994) ilustran este tipo de construcción de foco en cuatro idiomas diferentes: inglés, italiano, francés y japonés. Las oraciones representan una respuesta prototípica en cada idioma con respecto a la pregunta ¿Cómo está tu coche?, a través del cual se establece mi coche como el tópico.
Q: ¿Cómo está tu coche?
a. My car/it broke down.
Inglés
b. (La mia macchina) si è rotta. Italiano
c. (Ma voiture) elle est en panne. Francés
d. (Kuruma wa) koshooshita. Japonés

En cada caso, el predicado es un comentario o afirmación sobre el tópico mi coche. En inglés e italiano, el sujeto es también el tópico. En francés, el tópico es un sustantivo aislado, y, en japonés es un sujeto marcado con el morfema wa. En cada una de estas lenguas, el orden de los constituyentes es SV y hay un acento focal en el verbo.

En cambio, en ZAI se realiza el foco de predicado de manera distinta. Las construcciones de foco de predicado son de verbo inicial. El predicado ocupa la posición inicial de la cláusula en ZAI, seguida del sujeto-tópico ${ }^{3}$ :

3 A lo largo de este trabajo, las transcripciones y glosas en ZAI se escriben usando la ortografía oficial de ZAI (Alfabeto popular para la escritura del zapoteco del Istmo 1956; véase también Pickett 1979). Esta ortografía sigue generalmente las convenciones ortográficas del español mexicano. Como en el español, g y gu son /g/, hu es /w/, qu es /k/, gu es /gw/, y ch es /t $\mathrm{f} /$. Las excepciones a esto son las siguientes: $d x$ es /dz/; $x h$ es $/ \int /$; y $x$ es $/ 3 /$. Las vocales glotalizadas se indican mediante un apóstrofe [ '] inmediatamente después de la vocal y las vocales laringealizadas se muestran usando dos vocales consecutivas, [VV] (todavía dentro de una sola sílaba). Aunque el ZAI es 
(2) guxhiiñe xcoché,

gu-xhiiñe' $\quad \mathrm{x}=$ coche $=\mathrm{e}^{\mathrm{H}}$

COMPL-descomponerse POSS-coche $=1 \mathrm{SG}$

'Mi coche se descompuso'

En (3) se muestra un segundo ejemplo de la construcción de foco de predicado prototípico en ZAI:

(3) Q: (¿Qué hizo el niño?)

$\begin{array}{lll}\text { bidxaagabe } & \text { tí } & \text { dxaapahuiini' } \\ \text { bi-dxaaga=be } & \text { ti } & \text { dxaapa-huiini' } \\ \text { COMPL-encontrarse=3.HUM } & \text { uno niña-DIM } \\ \text { 'Él encontró una niña' } & \end{array}$

Esta es una cláusula transitiva donde el sujeto-tópico, el niño, aparece como un enclítico en el verbo, y el predicado, encuentra una niña, es el comentario o afirmación sobre el sujeto-tópico. De nuevo, se trata de una construcción de verbo inicial.

El verbo y el objeto están en el dominio de foco en este caso, pero ninguno recibe marcación focal en forma de un acento. ${ }^{4}$ Hay un tendencia descendente y gradual (downdrift) en el tono desde el comienzo de la cláusula hasta el final, pero no se produce un acento específico en el verbo o el objeto. El tono alto en la cláusula aparece en $t i$ como resultado del tono flotante del clítico de tercera persona $=b e$. Esto puede observarse en la pista de tono de este enunciado que se muestra a continuación en la Figura 1.

En general, los elementos que aparecen al comienzo de la unidad entonacional se pronuncian con una duración más larga, un registro de tono más alto y un rango de tono más amplio, es decir, propiedades asociadas con los inicios y las terminaciones de las unidades entonacionales. En este caso, el constituyente verbal aparece en la posición prosódicamente más prominente, al comienzo de la

un lenguaje tonal, el tono no está marcado en la ortografía oficial. Abordo esta problemática de dos maneras. Primero, debido a que los tonos superficiales se derivan directamente de los tonos subyacentes (Pérez Báez et al. 2015), se anotan los tonos subyacentes en el morfema usando la siguiente notación: $\left[{ }^{\mathrm{LH}}\right.$ ] para tono ascendente y [ $\left.{ }^{\mathrm{H}}\right]$ para tono alto. El tono bajo no se marca. Asimismo, en la transcripción del habla se anota también la información tonal usando la siguiente notación: [ `] para tono ascendente, [ ' $]$ para tono alto. El tono bajo no se marca.

4 Véase Lillehaugen (2008) en donde se muestra que en el zapoteco del Valle se usa un patrón de entonación específico para marcar la información topical. 


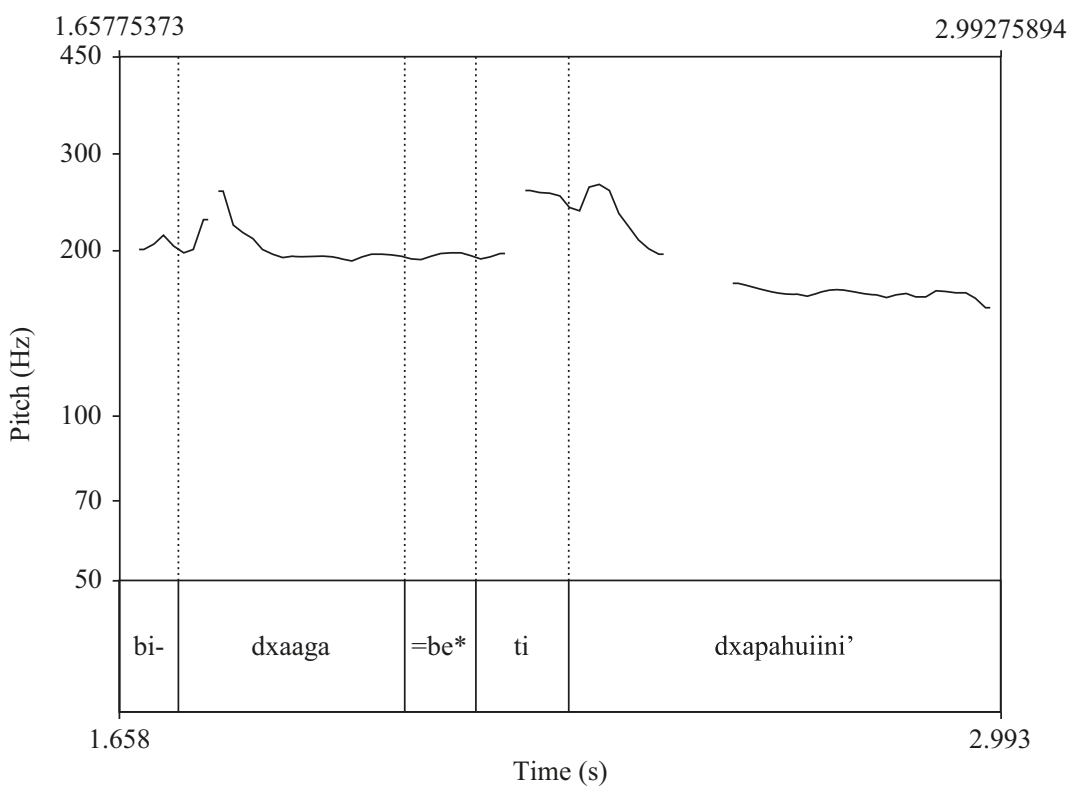

Figura 1: bidxaagabe ti dxaapahuiini'.

unidad entonacional. El objeto aparece en la siguiente posición más prominente desde el punto de vista prosódico; es decir, al final de la unidad entonacional.

Se puede considerar, ahora, el siguiente ejemplo, tomado de conversación:

(4) a. bibane'

bi-bani $=\mathrm{a}^{, \mathrm{H}}$

lá,

COMPL-despertar $=1 \mathrm{SG}$ LA

'Desperté,'

b. guzé $\quad x a$

gu-zi=a, ${ }^{\text {H }} \quad$ xa

COMPL-bañarse $=1 \mathrm{SG}$ INTJ

'me bañé,'

c. güé

ti jŭgo de narănjasi xá

gü-e-a, ${ }^{\text {LH }}$

ti $\mathrm{ju}^{\mathrm{LH}}$ go de nara ${ }^{\mathrm{LH}}$ nja-si ${ }^{\mathrm{LH}} \quad$ xa

COMPL-beber=1SG un jugo de naranja-solamente INTJ

'bebí solamente un jugo de naranja.' 
Aquí, el hablante recuerda y habla sobre los eventos de una rutina matutina. Cada una de las tres líneas es una construcción de foco de predicado. Cada cláusula es de verbo inicial, con el narrador como el sujeto-tópico, y cada predicado avanza los eventos de la narración.

Como se ve a continuación, en este caso tampoco hay acento de tono debido a la estructura focal y que esté asociado con ninguno de los constituyentes de la oración, como se ve en la Figura 2:

20120318_C_TVA_02_gue_ti jugo

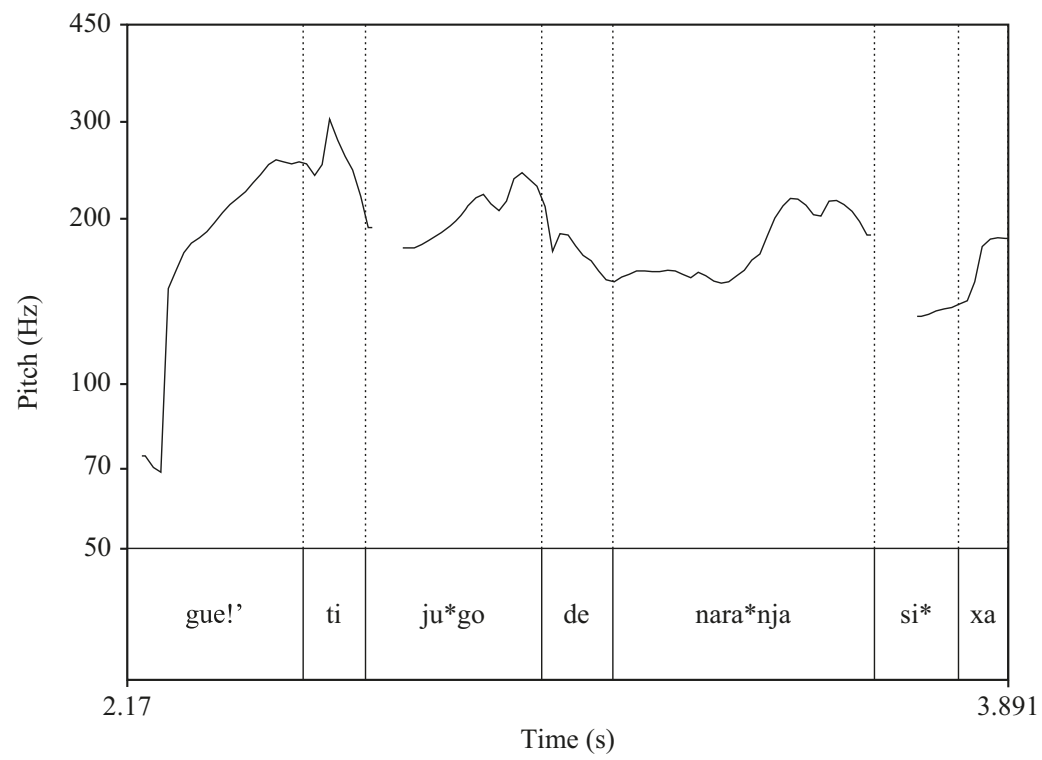

Figura 2: güé ti jŭgo de narănjasi xá.

En la última línea (4c), los tonos alto y ascendente se pueden atribuir directamente a los tonos subyacentes. El verbo güe tiene un tono alto proveniente del clítico de primera persona. Los sustantivos jugo y narănja tienen un tono ascendente en la sílaba acentuada, como es característico de muchos préstamos del español. Finalmente, la partícula -si unida al objeto contiene un tono alto flotante que aparece en la partícula final $x a$.

La principal característica de las construcciones de foco de predicado en ZAI, por lo tanto, es que emplean una cláusula principal de verbo inicial. El verbo es parte del dominio de foco y no recibe acento focal. Además, hay un downdrift gradual en el tono desde el principio de la cláusula hasta el final, pero no se produce un acento específico sobre el objeto. 


\subsection{Foco oracional}

En los casos de foco oracional no hay tópico y el dominio de foco es la totalidad de la oración (ejemplos de Lambrecht 1994):

(5) Q: ¿Qué pasó?
a. My car broke down.
Inglés
Lit. 'Mi coche se descompuso'
b. Mi si è rotta la macchina.
Italiano
Lit. 'Se me descompuso el coche'
c. J'ai ma voiture qui est en panne.
Francés
Lit. 'Tengo un coche que se descompuso'
d. Kuruma ga koshooshita.
Japonés
Lit. 'El coche se descompuso'

A diferencia de los ejemplos de foco de predicado presentados en (1), cada una de las oraciones en (5) carece de tópico. En cambio, se afirma toda la oración. El inglés usa la misma construcción sintáctica que en (1), sin embargo, en este caso, el sujeto recibe el acento focal. En italiano, el acento focal recae en el constituyente final de la oración, pero la construcción sintáctica se altera para que el sujeto aparezca en posición final. En francés, tanto el acento focal como la construcción sintáctica difieren de (1) y una parte de la información ahora se comunica a través de una cláusula relativa. En japonés, tanto el sujeto como el verbo reciben acento focal y el sujeto se marca usando el morfema ga en lugar de wa.

En ZAI, la construcción de foco oracional es formalmente idéntica a la construcción de foco de predicado en (2), excepto que en este caso no hay opción para representar al sujeto como un clítico. Necesariamente aparece como un sujeto léxico (o "fuerte", cf. Belloro y Guerrero 2012, Castillo Hernández 2014):
(6) guxhiiñe
xcoché,
gu-xhiiñe'
$\mathrm{x}=$ coche $=\mathrm{e}^{, \mathrm{H}}$
COMPL-descomponerse POSS-coche $=1 \mathrm{SG}$
'Mi coche se descompuso'

El uso más común de la construcción de foco oracional es en las construcciones presentativas, empleadas para introducir a los nuevos participantes en un discurso. En (7) se muestra un ejemplo de una construcción presentativa tomada de una narración: 

(7) bihuinni ti rígola
bi-huinni ti $\mathrm{ri}^{\mathrm{H}}$ gola
COMPL-aparecer un hombre
'Apareció un hombre'

En un uso típico como este, el narrador usa una construcción de foco oracional para introducir a un participante en el discurso. Al igual que con el foco de predicado, ésta es también una construcción de verbo inicial en la que el verbo toma la posición prosódica más prominente. El sujeto intransitivo se introduce como un sustantivo indefinido y ocupa la posición al final de la unidad entonacional. No hay un tópico, y el dominio de foco es la oración completa. No hay un acento especial asociado con esta construcción.

\subsection{Foco estrecho}

Mientras que el foco de predicado y el foco oracional son tipos de foco amplio, en casos de foco estrecho, el dominio de foco es un solo constituyente, que puede ser un objeto, sujeto, adjunto o incluso un verbo (los ejemplos son de Lambrecht 1994).

(8) Q: Escuché que tu moto se descompuso.

a. My car broke down

Lit. 'Mi coche se descompuso'

b. Si è rotta la mia macchina.

Italiano

Lit. 'Se ha descompuesto mi coche'

b’. È la mia macchina che si è rotta.

Lit. 'Es mi coche el que se ha descompuesto'

c. C'est ma voiture qui est en panne. Francés

Lit. 'Es mi coche el que se ha descompuesto'

d. Kuruma ga koshooshita.

Inglés

Lit. 'El coche se descompuso'

Japonés

En estas oraciones, el dominio de foco está restringido al sustantivo que denota coche en cada lengua. La presuposición es que "x se descompuso" y la afirmación es que fue el automóvil del hablante y no otra cosa. El inglés nuevamente usa la construcción sintáctica SV y, al igual que en (5a), el sujeto recibe el acento focal. En italiano, la construcción sintáctica se altera para que el acento focal recaiga de nuevo sobre el constituyente final de la oración. En 
francés, tanto el acento focal como la construcción sintáctica difieren de (1c) y (5c), y una parte de la información se comunica a través de una cláusula relativa. En japonés, el sujeto se marca usando el morfema $g a$, sin embargo, en (8d) solo el sujeto recibe acento focal.

En ZAI, en las construcciones de foco estrecho es posible que el sustantivo en foco aparezca después del verbo, pero esto es muy poco común, y en el orden preferido el constituyente en foco aparece antepuesto al verbo en la posición inicial de la cláusula:
(9) xcoché guxhiiñe
$\mathrm{x}=$ coche $=\mathrm{e}^{\text {, }} \quad$ gu-xhiiñe'
POSS-coche=1SG COMPL-descomponerse
'MI COCHE se descompuso'

En (10), se muestra un ejemplo tomado de una conversación:

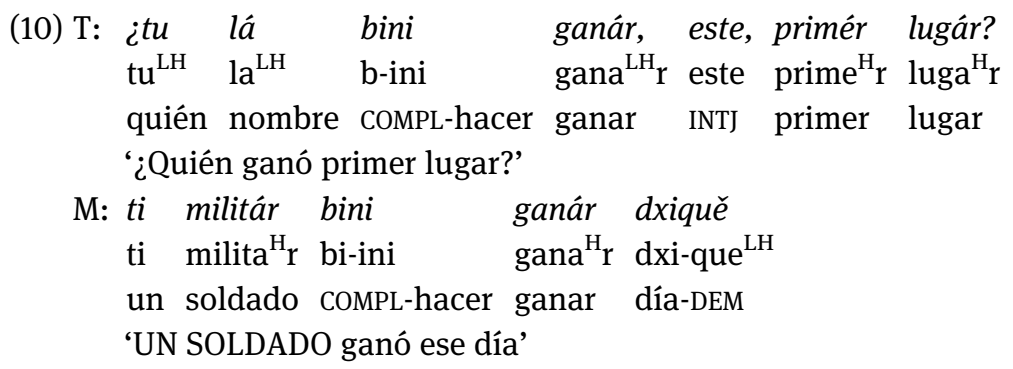

La pregunta del locutor T introduce la presuposición "x ganó el primer lugar." El locutor $M$ responde con la afirmación " $x$ es un soldado" y usa una construcción en la cual el sujeto aparece en posición pre-verbal seguido por el verbo que forma parte de la presuposición. La posición prosódica más prominente está ocupada en este caso por el sujeto.

En el siguiente ejemplo, la construcción de foco de predicado por parte del hablante en (11a) establece una presuposición que el hablante repite en (11b) mediante otra construcción de foco estrecho, esta vez sobre el objeto:

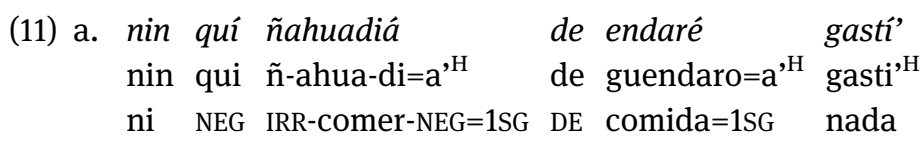

'No comí nada de mi comida' 
b. jŭgo quesí güé’

$\mathrm{ju}^{\mathrm{LH}}$ go que ${ }^{\mathrm{LH}}-\mathrm{si}^{\mathrm{LH}} \quad$ gu-e $=\mathrm{a}^{, \mathrm{H}}$

jugo DEM-solamente COMPL-beber $=1 \mathrm{SG}$

'Solamente EL JUGO bebí.'

El verbo para comer/beber es el mismo en (11a) y (11b) (la forma fonológica del verbo está condicionada por el prefijo aspectual). En (11a), el hablante establece la presuposición "comí/bebí x". Continúa en (11b) con la afirmación "x es solo el jugo".

En estos casos, no es el verbo sino un sustantivo el que se encuentra en la posición prosódica prominente al comienzo de la unidad entonacional. Sin embargo, al igual que en los ejemplos anteriores, no hay un acento particular asociado con alguna parte del enunciado, como se observa en la Figura 3:

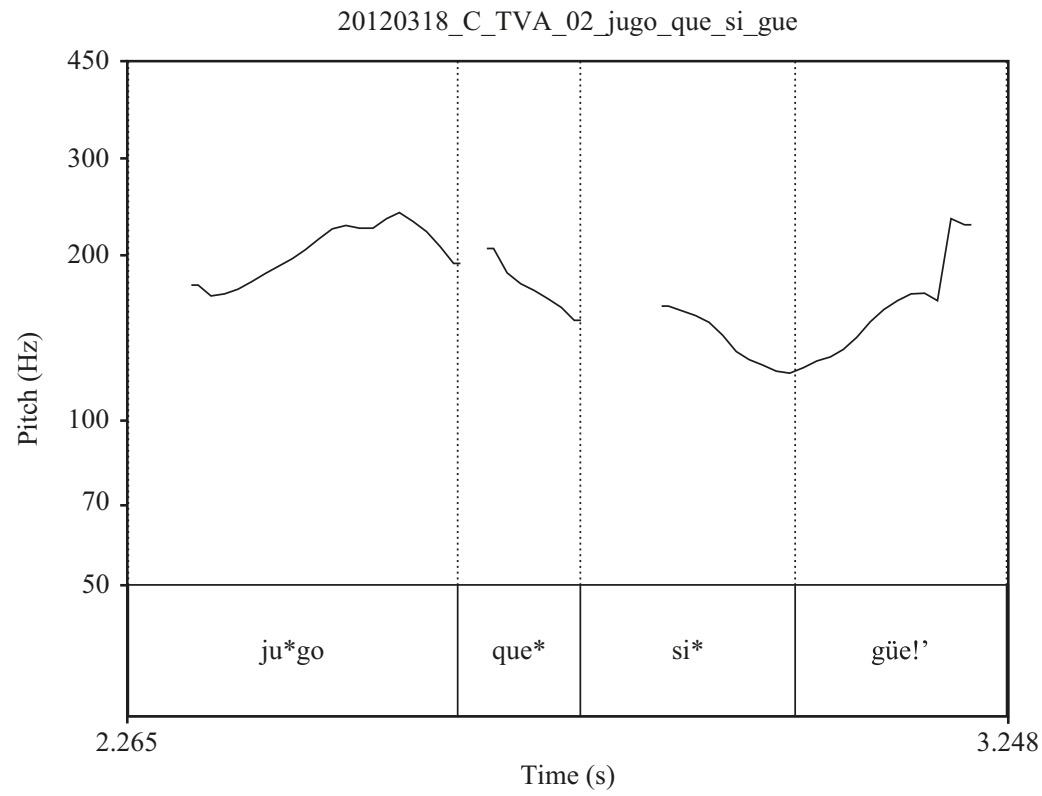

Figura 3: jŭgo quesí güé’.

Se puede comparar esta construcción con la construcción de foco de predicado, güe ti jugo de naranjasi $x a$ en (4) pronunciado por el mismo hablante. Las construcciones tienen un contenido proposicional casi idéntico, excepto que en (4) el hablante usa un objeto indefinido y en (11) usa un objeto definido. Los dos enunciados difieren también en el orden de los constituyentes, dado que el 
objeto ocurre en posición pre-verbal en la construcción de foco estrecho en (4) y en posición posverbal en la construcción de foco de predicado en (11). Se retornará a otros pares de enunciados como estos en la Sección 3, donde se muestra el uso de foco de predicado en la conversación seguido por el foco estrecho y se explora la función discursiva combinada de estos dos tipos de construcciones.

Sin embargo, debe tenerse en cuenta que las construcciones de foco estrecho no tienen que ser de argumento inicial. Una construcción como la siguiente, con una estructura de verbo inicial, también sería aceptable en la misma situación:

$\begin{array}{lll}\text { güé } & \text { jǔgo } & \text { quesí } \\ \text { gu-e=a, } & \mathrm{ju}^{\mathrm{LH}} \text { go } & \text { que }^{\mathrm{LH}}-\mathrm{Si}^{\mathrm{LH}} \\ \text { COMPL-beber=1SG jugo } & \text { DEM-solamente } \\ \text { 'Bebí solamente EL JUGO.' }\end{array}$

No existe una marca formal que separe esta construcción de una construcción de foco de predicado, dejándola formalmente ambigua. Aunque un sustantivo en posición preverbal señala la naturaleza focal del sustantivo, en construcciones de verbo inicial, el foco puede caer sobre el verbo. Solamente la información contextual permite a los participantes comprender que la presuposición y la afirmación siguen siendo las mismas que en la construcción original en (11b). Aun así, mientras que una estructura de verbo inicial se puede usar alternativamente en una construcción de foco de predicado así como en una construcción de foco estrecho, salvo en algunos casos, el uso de un constituyente preverbal señala una construcción de foco estrecho. ${ }^{5}$

\subsection{Resumen de la estructura focal del ZAI}

En resumen, en esta sección se ha observado el siguiente patrón en la estructura informativa de ZAI: mientras que las construcciones de foco oracional $\mathrm{y}$ foco de predicado son consistentemente de verbo inicial, las construcciones de foco estrecho pueden ser de verbo inicial o, más comúnmente, pueden tener

5 En el ZAI existe una construcción de topicalización en la que un sujeto transitivo topical aparece en posición inicial de cláusula seguido por el verbo y el objeto. En estas construcciones es obligatoria la marca de sujeto en el verbo en forma de clítico para diferenciar esta construcción de una construcción de foco estrecho. Para un análisis más detallado sobre estas construcciones de topicalización en el ZAI, véase Bueno Holle (2016, 100-102). 
constituyentes preverbales (dentro de la cláusula). Es decir, el orden de constituyentes en ZAI se adapta a las funciones del discurso.

En contraste con los datos reportados del zapoteco del Valle (Lillehaugen 2008), en el ZAI no hay evidencia de un acento directamente asociado con el tópico ni el foco. Sin embargo, los elementos pueden mostrar varias propiedades prosódicas: mayor duración, mayor registro de tono y mayor rango de tono, que pueden estar relacionadas con la posición dentro de la unidad entonacional en la que aparecen. Los elementos en foco (ya sean constituyentes nominales o verbales) tienden a ocurrir en posiciones prosódicamente más prominentes; es decir, a comienzos de unidades entonacionales. Los elementos que aparecen al comienzo de las unidades entonacionales se pronuncian con una duración más larga, un registro de tono más alto y un rango de tono más amplio; es decir, propiedades asociadas con los comienzos de las unidades entonacionales.

\subsection{La tipología de estructuras focales de Van Valin (1999)}

Basándose en la conceptualización de estructura focal propuesta por Lambrecht (1994) revisada en la sección anterior, Van Valin (1999) propone una manera de comparar y clasificar las lenguas.

Las lenguas varían enormemente en cuanto a los recursos lingüísticos que emplean para llevar a cabo diversas funciones del discurso. Para Van Valin, una de las dimensiones en las que pueden variar es la dimensión sintáctica, ya que las lenguas pueden ser más o menos rígidas en términos de la organización sintáctica de los constituyentes. Como muestran los ejemplos en la sección anterior, el inglés, por ejemplo, tiene una sintaxis rígida, así como el francés, mientras que el italiano tiene una sintaxis flexible. Una segunda dimensión es la del dominio focal, por lo cual las lenguas pueden ser más o menos rígidas en términos de dónde se encuentra el dominio focal dentro de una cláusula dada.

El uso de Van Valin de la noción de "dominio potencial de foco" es fundamental para su análisis de la estructura de foco como relativamente rígido o flexible. Van Valin $(1999,513)$ define el "dominio potencial de foco" como "la parte de la oración en la que potencialmente se puede encontrar un elemento focal.” En inglés, por ejemplo, el dominio potencial de foco es la cláusula principal, lo cual significa que el acento focal potencialmente puede caer sobre cualquier constituyente dentro de la cláusula principal, ya sea el predicado o el final de una cláusula (e.g. (1a)), o sobre un sujeto preverbal (e.g. (5a), (8a)). Por lo tanto, se dice que el inglés es un ejemplo de un idioma con un dominio potencial de foco flexible. 
La clasificación de las lenguas en las dos dimensiones, rígida o flexible, por un lado y, por el otro, la estructura de sintaxis y la estructura focal proporciona, siguiendo a Van Valin, el siguiente marco tipológico (Tabla 1):

Tabla 1: Una tipología de estructura focal (Van Valin 1999).

\begin{tabular}{lll}
\hline & $\begin{array}{l}\text { Estructura focal } \\
\text { rígida }\end{array}$ & $\begin{array}{l}\text { Estructura focal } \\
\text { flexible }\end{array}$ \\
\hline Sintaxis rígida & Francés & Inglés \\
Sintaxis flexible & Italiano & Ruso \\
\hline
\end{tabular}

Esta forma de clasificar las lenguas se basa en si el orden de los constituyentes en las cláusulas principales depende de principios sintácticos (por ejemplo, de las relaciones gramaticales) o de principios pragmáticos (por ejemplo, del estado cognitivo de los referentes). Por un lado, el orden de constituyentes puede estar restringido por principios pragmáticos. Por ejemplo, la gramática de una lengua puede prohibir la asignación de foco a sujetos preverbales, como en italiano, o puede reservar una posición sintáctica específica para información particularmente “de interés”, como en Cayuga (Mithun 1992). Es decir, el dominio de asignación de foco puede ser más o menos fijo (típicamente con respecto al verbo). Por otro lado, en aquellas lenguas en las que el orden de los constituyentes se ve más estrechamente restringido por principios sintácticos, como en el inglés, la codificación de la estructura informativa se lleva a cabo con frecuencia exclusivamente por medios prosódicos, dejando el orden de constituyentes intacto.

Dado que la distinción entre rígido y flexible debe entenderse como un continuo más que como una distinción binaria, pasaremos ahora a analizar el potencial dominio de foco del ZAI en el continuo entre la rigidez a la flexibilidad para, de manera más general, ubicar la estructura focal del ZAI en de la tipología de Van Valin. ${ }^{6}$

En términos de estructura focal, el potencial dominio de foco en ZAI es relativamente flexible, dado que los constituyentes en foco pueden aparecer en posición preverbal o posverbal. Mientras que en construcciones de foco amplio el dominio de foco es posverbal, en construcciones de foco estrecho hay una

6 Para una investigación preliminar en esta línea sobre el zapoteco del Valle, véase Castillo Hernández (2014). En ese trabajo, Castillo Hernández analiza el zapoteco del Valle como una lengua pragmáticamente flexible y con sintaxis rígida. 
fuerte preferencia por que los constituyentes en foco aparezcan pre-verbalmente. También son posibles los constituyentes posverbales en foco, incluyendo objetos transitivos e intransitivos y sujetos intransitivos (los sujetos transitivos en foco deben aparecer en posición preverbal). Los sustantivos plenos y los pronombres independientes, ya sea en posición preverbal o posverbal, generalmente son parte del dominio de foco. En contraste, como se esperaría, los clíticos pronominales siempre son tópicos.

En términos de la sintaxis, el ZAI también es relativamente flexible, ya que varios elementos pueden ocurrir antes o después del verbo, lo cual muchas veces está determinado por las necesidades de la estructura de foco. Esto indica, por lo tanto, que la estructura de foco es más rígida que la sintaxis, ya que la estructura de foco puede motivar ciertas construcciones sintácticas, mientras que lo inverso raramente se cumple, es decir, la estructura sintáctica no motiva cambios en el dominio de foco.

Aunque la marcación de foco en ZAI no implica el uso de un acento, cualquier constituyente en foco debe aparecer al principio o al final de una unidad entonacional, es decir, en posición de prominencia prosódica. Las estructuras de verbo inicial, donde el verbo aparece en la posición prosódica más prominente, tienden a ser aquellas en las que el verbo forma parte de la afirmación. En las estructuras sin verbo inicial, donde los elementos no verbales ocupan la posición prosódica más prominente, el verbo forma parte de la presuposición. En otras palabras, el elemento inicial de la cláusula, ya sea verbal o no verbal, suele formar parte del dominio de foco, y coincide con la prominencia prosódica de la frase. ${ }^{7}$

\section{La estructura focal en uso: las construcciones quiásticas}

En la Sección 2, se revisaron los diversos tipos de construcciones de foco disponibles para los hablantes de ZAI. Se vieron varias formas en que los hablantes explotan diversas combinaciones de frases nominales

\footnotetext{
7 En la Sección 4 se mostrará que una de las funciones del morfema LA es marcar el final de una unidad entonacional separada, entre otras cosas, para dar prominencia prosódica a la frase. Una posible motivación, por lo tanto, para la gama de órdenes de constituyentes observada en los diversos tipos de construcciones en ZAI, así como la distinción entre tipos de foco amplio y estrecho, podría ser prosódica. Véase Bueno Holle (2019, 87-103) para sugerencias adicionales a favor de esta línea de investigación.
} 
y de órdenes de constituyentes para lograr sus objetivos discursivos. En esta sección, ${ }^{8}$ se busca ampliar esta perspectiva mediante el análisis de una estrategia conversacional de los hablantes de ZAI, en el que se emplea el uso paralelo y quiástico del foco de predicado y el foco estrecho para cumplir objetivos conversacionales específicos.

En el siguiente ejemplo, el hablante relata algo que comió la noche antes de un evento importante en su vida. Él explica cómo tenía hambre esa noche y comió como lo haría normalmente:

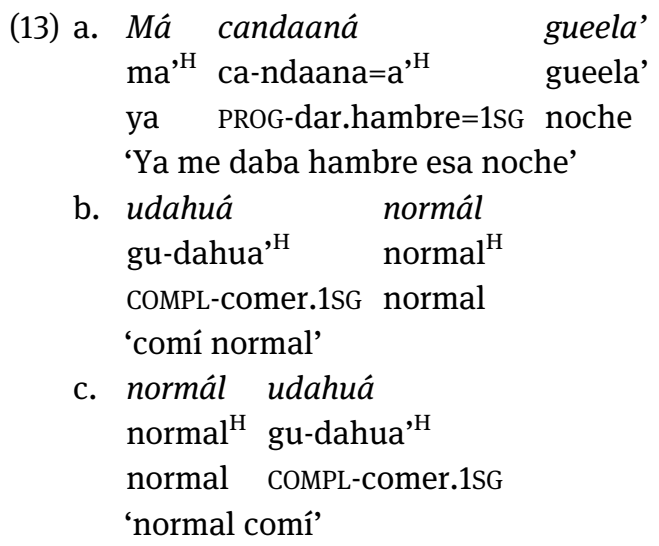

El hablante menciona en (13a) que se sentía hambriento esa noche. En (13b) utiliza una construcción de foco de predicado en la que afirma que comió como lo haría normalmente, udahuá normál. Termina su turno en (13c) con una construcción de foco estrecho, normál udahuá', una construcción espejo del enunciado en (13b). En términos pragmáticos, sin embargo, hay poco que (13c) agregue a la comprensión del evento por parte del oyente dado que se trata del mismo contenido proposicional. La información de que el hablante comió como lo haría normalmente esa noche ya ha sido transmitida.

No hay acento de tono adicional asociado con ninguna parte de cualquiera de las emisiones, lo cual se observa en la pista de tono que se muestra a continuación en la Figura 4. También se puede ver que no hay una pausa entre (13b) y (13c):

El uso de la construcción de foco predicado seguido inmediatamente por una construcción de foco estrecho puede conceptualizarse como una estructura discursiva propia que explota el "paralelismo" (Jakobson 1966, Fox 1977).

8 Esta sección está adaptada y traducida de Bueno Holle (2019, 103-107). 


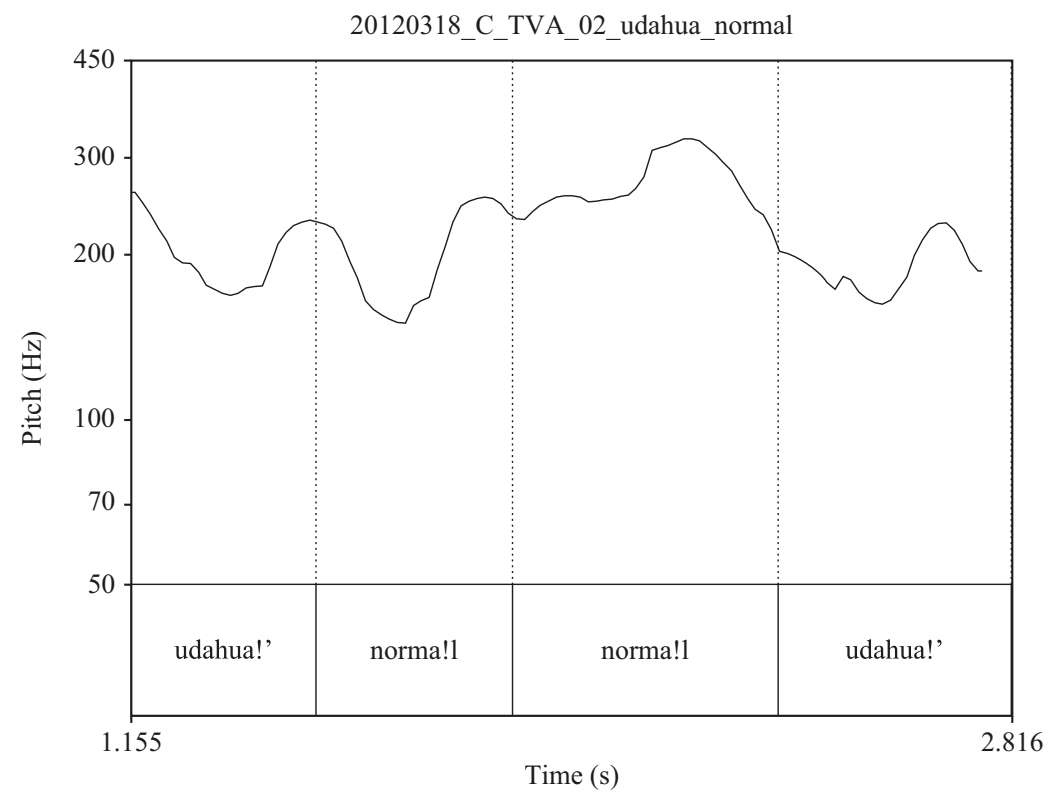

Figura 4: udahuá normál, normál udahuá.

Una de las funciones de este paralelismo o "estructura quiástica" (Silverstein 1984), es ayudar al hablante a extender su turno de habla por una unidad entonacional adicional. Al mismo tiempo, la combinación de foco de predicado más foco estrecho juntos marcan el final del turno del hablante. El hablante cede la palabra y el uso de la estructura quiástica une las dos unidades entonacionales para ser interpretadas juntas.

Este uso combinado de foco de predicado más foco estrecho como una estructura quiástica se emplea a menudo en la conversación entre hablantes de ZAI. A continuación se presenta un segundo ejemplo. Aquí, el hablante menciona su participación en un maratón internacional en la Ciudad de México 25 años antes y utiliza la estructura quiástica en las líneas (14b-14c) para resaltar su edad joven en ese momento:

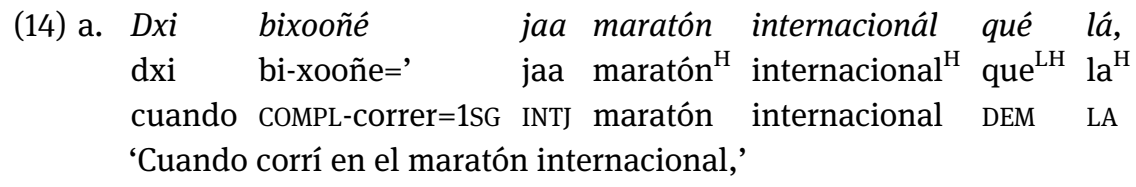


b. veintidós iza napá dxiquě

veintido $^{\mathrm{LH}} \mathrm{S}$ iza $\mathrm{n}$-apa $=\mathrm{a}^{, \mathrm{H}} \quad \mathrm{dxique}^{\mathrm{LH}}$

veintidos año HAB-tener=1SG entonces

'VEINTIDOS años tenía en ese entonces'

c. má napá veintidós iza

ma ${ }^{\text {,H }} \mathrm{n}$-apa $=\mathrm{a}^{, \mathrm{H}} \quad$ veintido $^{\mathrm{LH}} \mathrm{S}$ iza

ya HAB-tener=1SG veintidos año

'tenía veintidós años.'

Luego de comenzar su turno con una frase adverbial marcada con LA en (14a) que presenta el evento del maratón internacional como tópico, el hablante usa una construcción de foco estrecho en (14b) para comentar sobre su edad en ese momento. En (14c), el hablante repite un enunciado equivalente, esta vez usando una construcción de verbo inicial, pero de nuevo de foco estrecho y en la cual su edad aparece esta vez pospuesta al verbo.

En un último ejemplo, también de una conversación, se observa un uso similar de la estructura paralela y quiástica. En las primeras líneas, T le pregunta a C qué tipo de cultivos solía cultivar su padre en su parcela de tierra y si tenía ganado:

(15) a. T: ¿Xi bídxíbabe yá'?

$\mathrm{xi}^{\mathrm{LH}}$ bi-dxi'H ${ }^{{ }^{\mathrm{H}}} \mathrm{ba}=\mathrm{be}^{\mathrm{LH}} \quad$ ya'

qué COMPL-cultivar=3.HUM Q

¿¿Qué cultivó?'

b. C: Bidxíbabe pǔru xubá'

bi-dxi, ${ }^{\mathrm{H}}$ ba $=\mathrm{be}^{\mathrm{LH}} \quad \mathrm{pu}^{\mathrm{LH}} \mathrm{ru} \quad \mathrm{xuba}{ }^{\mathrm{H}}$

COMPL-cultivar=3.HUM solamente maíz

'Sólo cultivó maíz'

c. T: ¿Gupabe

gu-apa=be ${ }^{\mathrm{LH}} \quad \mathrm{yu}^{\mathrm{LH}} \mathrm{ze}^{\mathrm{LH}} \mathrm{la}^{\mathrm{H}}$

COMPL-tener=3.HUM ganado $\mathrm{Q}$

‘Tenía ganado?'

d. C: pǔru ngă ngá rudxíbacabě

$\mathrm{pu}^{\mathrm{LH}} \mathrm{ru} \mathrm{nga}^{\mathrm{LH}} \mathrm{nga}^{\mathrm{H}}$ ru-dxi ${ }^{\mathrm{H}} \mathrm{ba}=\mathrm{ca}=\mathrm{be}$

solamente DEM NGA HAB-cultivar $=\mathrm{PL}=3$. HUM

'Sólo eso cultivan'

e. purtí cheri lá,

purti $^{\mathrm{H}}$ cheri $^{\mathrm{LH}} \mathrm{la}^{\mathrm{H}}$

porque aquí LA

'Porque por aquí,' 


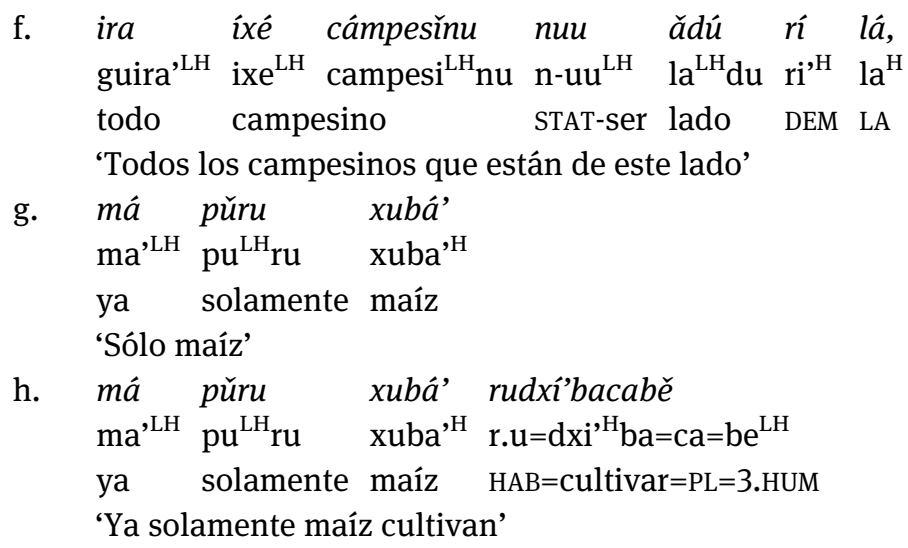

En respuesta a la pregunta inicial de $\mathrm{T}$, ¿Qué cultivó?, C responde en (15b) con una construcción de verbo inicial, diciendo que su padre solamente cultivó maíz. Luego, C continúa este pensamiento afirmando en (15d) que en esa región el maíz es lo único que se cultiva y lo hace usando una construcción de foco estrecho. Repite este pensamiento nuevamente en (15g) usando una cláusula sin verbo, 'sólo maíz'. Finalmente, termina su turno en (15h) con una construcción de foco estrecho (má pǔru xubá' rudxí'bacabě) que es una imagen espejo de su enunciado de verbo inicial en (15b) (bidxíbabe pǔru xubá) con la que se abrió el tema de conversación.

El uso de la construcción de foco de predicado seguido inmediatamente por la construcción de foco estrecho explota el paralelismo de las estructuras sintácticas e informativas de ambas. Al usar esta estructura paralela y quiástica, las dos unidades entonacionales se unen para interpretarse juntas, y el hablante amplía su turno de habla por una unidad entonacional adicional, con la segunda parte, la construcción del foco estrecho, marcando el final del turno del hablante y cediendo así la palabra.

\section{Los usos de la partícula $L A$}

Uno de los rasgos comunes de las lenguas zapotecas es la presencia de un morfema LA que se caracteriza por tener funciones discursivas específicas. ${ }^{9}$

9 Esta sección está adaptada y traducida del capítulo 6 de Bueno Holle (2019, 118-125). Véase Riggs y Marlett (2010) para un esbozo general sobre algunos rasgos sintácticos y discursivos de este morfema en una amplia gama de lenguas zapotecas. Este morfema lleva una forma 
Por ejemplo, en el zapoteco de San Lucas Quiaviní (Munro y Lopez 1999, Lee 1997, 2000) LA se emplea en la marcación de foco, y en el zapoteco de Tlacolula (Lillehaugen 2008) se usa en la marcación de objetos animados. ${ }^{10}$ En cambio, en el ZAI, el morfema LA nunca se usa con elementos con función de foco:

$$
\begin{aligned}
& \text { (16) A: ¿Tu bíninǐ? } \\
& \mathrm{tu}^{\mathrm{LH}} \text { bi-uni=ni }{ }^{\mathrm{LH}} \\
& \text { quién COMPL-hacer }=3 \text { SG.INAN } \\
& \text { ¿Quién lo hizo? } \\
& \text { B: (*lá) Tomăs bi’ninǐ } \\
& \mathrm{Toma}^{\mathrm{LH}} \mathrm{S} \text { bi-uni=ni }{ }^{\mathrm{LH}} \\
& \text { Tomás COMPL-hacer=3SG.INAN } \\
& \text { '(^la) Tomás lo hizo (*la)' }
\end{aligned}
$$

En esta sección, se revisarán los tipos de construcciones en que los hablantes del ZAI utilizan LA, incluyendo al final de las cláusulas adverbiales y condicionales, y se verá la manera en que se emplea, no para la marcación de foco, sino para “fijar la escena”, o como scene-setting topic (Lambrecht 1994, 125). Esta discusión hará posible, al final de esta sección, considerar la manera en que los hablantes del ZAI utilizan la partícula LA en el discurso y la conversación y con qué funciones.

\subsection{La partícula LA y la información topical}

En el ZAI, el morfema LA es un enclítico que lleva un tono alto e invariablemente aparece al final de una unidad entonacional, seguido de una pausa (y nunca en otra posición). ${ }^{11}$ En uno de sus usos más comunes, SE emplea al final de

\footnotetext{
distinta según la variante del zapoteco, por ejemplo le'e en el zapoteco de Amatlán (Riggs 1987), làa' en el zapoteco del Valle (Lillehaugen 2008), laa en el zapoteco de Xanica (Piper 1995), le' en el zapoteco de Quioquitani (Ward 1987) o nna en el zapoteco de la Sierra Juárez (Gibbs 1977). Este morfema, que en este trabajo se glosará como LA, se distingue de otro morfema LAA relacionado con el sistema de pronombres, pero que en muchas variantes tiene la misma forma fonológica.

10 Riggs y Marlett (2010) reportan usos y funciones similares para la gran mayoría de lenguas zapotecas.

11 Esto se diferencia de la manera en que se emplea el morfema LA en otras variantes del zapoteco donde principalmente aparece antepuesto al sustantivo o a la frase (cf. Riggs y Marlett 2010, Appendix A).
} 
cláusulas temporales que avanzan o dan información sobre la secuencia de eventos en una narrativa, como en (17) y (18):

\begin{tabular}{|c|c|c|c|}
\hline ǒra & bidxiguetalube & bíiyabe & bádudxaapa \\
\hline $\mathrm{o}^{\mathrm{LH}} \mathrm{ra}$ & bi-dxiguetalu=be ${ }^{\mathrm{LH}}$ & bi-uuya $=b e^{\text {LH }}$ & badudxaapa \\
\hline cuando & COMPL-voltear=3SG.ANIM & COMPL-ver=3SG.ANIM & niña \\
\hline $\begin{array}{l}\text { que } \\
\text { que }^{\mathrm{LH}}\end{array}$ & $\begin{array}{l}\text { lá, } \\
\operatorname{la}^{\mathrm{H}}\end{array}$ & & \\
\hline
\end{tabular}

DIST LA

'Cuando volteó y vió a la niña LA,'

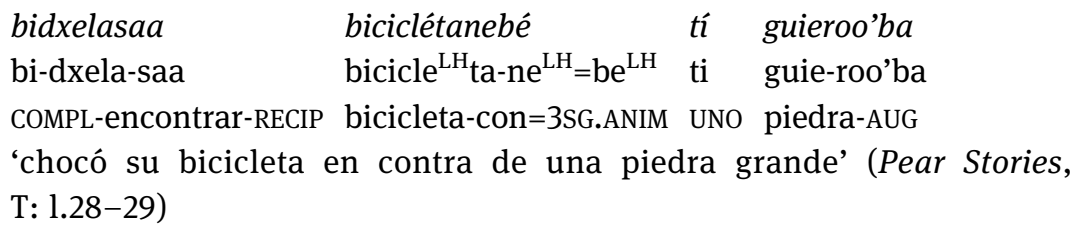

(18) raque má zeeda tí xcuídihuiini lá, raque $^{\mathrm{LH}}$ ma $^{\text {,H }}$ zeeda $^{\mathrm{LH}}$ ti xcui $^{\mathrm{H}}$ di-huiini $\mathrm{la}^{\mathrm{H}}$ luego ya PART.venir uno niño-DIM LA 'Luego al llegar un niño LA'

\begin{tabular}{|c|c|c|c|}
\hline & rá & cuchuugu pěrá & $q u e \check{~}$ \\
\hline bi-iya $=b e^{\mathrm{LH}}$ & ra & cu-chuugu' $=\varnothing \mathrm{pe}^{\mathrm{LH}} \mathrm{ra}$ & $q u e^{\mathrm{LH}}$ \\
\hline COMPL-ver=3SG.ANIM & cuando & PROG-cortar $=3$ pera & DEM \\
\hline
\end{tabular}

Tanto en (17) como en (18), LA aparece luego de una cláusula temporal y al final de la unidad entonacional. Este uso con cláusulas temporales es muy común y, a pesar del hecho de que los hablantes no lo consideran obligatorio, es raro encontrar casos en el habla espontánea en los que LA esté ausente.

La partícula LA también aparece consistentemente al final de la frase inicial de las cláusulas condicionales, como en (19):

(19) $\mathrm{Pa}$ guiába nisaguie guixí lá, qué ziaá' $\mathrm{pa}^{\mathrm{LH}}$ gui $^{\mathrm{LH}}$-aba nisa-guie guixi, ${ }^{\mathrm{H}} \mathrm{la}^{\mathrm{H}} \mathrm{que}^{\mathrm{H}} \mathrm{zi}^{\mathrm{LH}}-\mathrm{e}=\mathrm{a}^{, \mathrm{H}}$ si POT-caer agua-piedra mañana LA NEG FUT-ir=1SG 'Si llueve mañana LA, no voy' (Pickett et. al. 1998, 109)

El uso de LA con cláusulas condicionales es significativo porque se sabe que tanto las cláusulas adverbiales como las cláusulas condicionales están 
explícitamente marcadas en otras lenguas con la marca de tópico (véase Thompson et al. 2007, 292). Por ejemplo, en hua (Papuana), tanto las cláusulas condicionales como los tópicos (y los interrogativos) están marcados con ve (Haiman 1978). De forma similar, en turco, un sufijo condicional marca los tópicos (Kerslake 1996).

Sin embargo, las construcciones adverbiales y condicionales no son las únicas cláusulas que se suelen marcar usando la marcación de tópico. Por ejemplo, en chino, las cláusulas de concesión, de razón, de tiempo y de condición ocurren con las mismas partículas usadas en la marcación de tópico (Thompson et al. 2007, 293). En lisu (Burmano-Tibetano), las cláusulas adverbiales que funcionan como tópicos están marcadas de la misma forma que los sustantivos topicales, utilizando el morfema nya (Thompson et al., 2007, 294). En el kurdo central, las cláusulas temporales están marcadas como tópicos por una partícula aditiva (Öpengin 2013). En el karbi (Burmano-Tibetano), la partícula aditiva marca información contrastiva o sustantivos contrastivos (Konnerth 2013).

De hecho, la marcación de un contexto adverbial diferente al de la cláusula anterior es un uso extremadamente común de LA. A continuación se muestra un uso de este tipo, tomado de una conversación informal:

(20) a. Xagueté nisa runidxi binň̌
xaguete $^{\mathrm{H}}$ nisa ru-nidxi binni ${ }^{\mathrm{LH}}$
debajo agua HAB-bucear persona
'Debajo del agua la gente bucea'
b. ne lú nisa lá,
ne lu nisa la ${ }^{\mathrm{H}}$ lu
y cara agua LA
'y sobre el agua LA,'
c. rixuubacabě
ri-xuuba'=ca-be ${ }^{\mathrm{LH}}$
HAB-nadar=PL-3SG.ANIM
'nadan.'

Después de ofrecer una alternativa en (20a) a lo que las personas pueden hacer bajo el agua, el hablante utiliza otro modificador adverbial en (20b) para introducir lo que las personas pueden hacer por encima del agua. Es el contraste entre ‘debajo del agua' y 'sobre el agua' lo que está siendo marcado con LA. ${ }^{12}$

12 En esta función de contraste y de cohesión textual, el morfema LA del ZAI parece tener características similares al morfema BAA en somali reportado por Matić y Wedgwood (2013, 138-140). 
Hay varias razones por las que es común que las cláusulas adverbiales o condicionales desempeñen esta función de "fijar la escena" (scene-setting topic) para la cláusula matriz (Lambrecht 1994, 125). En primer lugar, su función principal es vincular la cláusula anterior con la cláusula a la que están adjuntas y, al mismo tiempo, establecer un marco dentro del cual se sostiene la siguiente predicación (Thompson et al 2007, 294). En segundo lugar, las cláusulas adverbiales o condicionales sirven para recapitular el material ya mencionado, es decir, para establecer un terreno común entre los interlocutores. Finalmente, a menudo hay un tono alto que aparece al final de la primera unidad entonacional, y que luego cae en la segunda. Esto ayuda a unir la información en una estructura que permite la interpretación en conjunto (cf. las construcciones quiásticas, Sección 3; también Sicoli 2007, 126-7).

De esta forma, las construcciones con LA forman parte de la presuposición pragmática (es decir, del tópico) que, como notan Thompson et al. (2007, 292), "establece un marco dentro del cual continuar con un discurso, de la misma manera que lo hace una pregunta.” De hecho, todas las construcciones que involucran LA revisadas arriba comparten una morfología común con las preguntas de sí/no, como se verá a continuación.

\subsection{Las preguntas de sí/no}

Las preguntas de sí/no en ZAI se forman mediante el uso de una partícula que tiene la misma forma de la partícula LA analizada en la sección anterior (también con tono alto $)^{13}$ :

\begin{tabular}{|c|c|}
\hline ¿Riuuladxu’ & Lulá \\
\hline ri=yuu-ladxi=lu' & Lula'H $^{\text {, }}$ \\
\hline $\begin{array}{l}\text { HAB }=\text { entrar-vientre }=2 S G \\
\text { 'Te gusta Oaxaca?' }\end{array}$ & Oaxaca \\
\hline
\end{tabular}

Hay tres razones principales para pensar que este es el mismo morfema que la partícula LA analizada en la sección anterior. En primer lugar, es poco común en las lenguas de verbo inicial que las partículas interrogativas ocurran en posición final de la cláusula (Payne 1990). En segundo lugar, se ha encontrado una

13 En el zapoteco del Valle esta partícula tiene otro tono (Munro et al. 2008). Sería relevante un estudio detallado sobre el morfema LA en otras variantes del zapoteco para investigar si existe una relación similar entre su uso como focalizador y su uso en preguntas. 
morfología común entre interrogativos y condicionales (Haiman 1978). Finalmente, se sabe que la marcación de cláusulas condicionales se desarrolla consistentemente a partir de partículas interrogativas (König y Siemund 2007, 296).

Una posible razón para la existencia de esta conexión en ZAI entre el uso de LA en las preguntas de sí/no y en contextos discursivos como los que se analizaron en los ejemplos (17)-(20) de la Sección 4.1, es que los hablantes de ZAI utilizan la partícula LA como un recurso en las interacciones para construir un contexto compartido. Más específicamente, LA se puede analizar como un ejemplo de "marcación de prueba" (try-marker; Sacks, Schegloff y Jefferson 1974). Sacks, Schegloff y Jefferson (1974) definen la "marcación de prueba" como la mención de información compartida entre los interlocutores con un contorno de entonación ascendente y seguida de una breve pausa, posiblemente buscando la confirmación del referente de otros participantes (Pekarek Doehler 2011).

Una forma de considerar esto es pensar que las oraciones marcadas con LA son similares a "mini-conversaciones" (Thompson et al., 2007, 292). Por ejemplo, la construcción en (22) es semánticamente similar a la construcción condicional mencionada anteriormente en (19):

(22) A: ¿Chi guiaba nisaguie guixí’ la? ‘¿Va a llover mañana?’

B: Ziaba 'Va a llover'

A: Que ziaá' 'No voy'

El locutor A usa una frase marcada con LA (similar a la prótasis en la correspondiente construcción condicional en (19)) para buscar la confirmación de B en forma de una respuesta sí/no. En este caso, la respuesta explícita de B proporciona un supuesto compartido dentro del cual A puede ahora proceder a comunicar el contenido proposicional principal (la apódosis en la construcción condicional correspondiente), es decir, que no irá. La construcción condicional tiene una función muy similar en la interacción, solo que no hay una respuesta explícita del oyente después de la prótasis.

El uso de LA con la función de construir un entorno de supuestos mutuos también se observa en casos en los que un hablante está construyendo una lista. Un ejemplo se da en (23), tomado de una conversación informal entre tres hombres adultos. La partícula LA se usa en (23b), (23d) y (23e):

(23) a. péru ti dxi ănte pe ${ }^{\mathrm{LH}} \mathrm{ru}$ ti dxi $\mathrm{a}^{\mathrm{LH}}$ nte pero uno día antes 'Pero un día antes' 
b. viěrne huaxhinni que lá, $v^{2} e^{\mathrm{LH}}$ rne huaxhinni que ${ }^{\mathrm{LH}} \mathrm{la}^{\mathrm{H}}$

viernes tarde DEM LA

'ese viernes por la tarde LA'

c. uxudxidǔ

$g u=x u d x i=d u^{\mathrm{LH}}$

COMPL $=$ emborracharse $=1$ PL.EXCL

'nos emborrachamos'

d. laabe lâ,

laa $=\mathrm{be}^{\mathrm{LH}} \quad \mathrm{la}^{\mathrm{H}}$

base $=3$ SG.ANIM LA

'él (apuntando) LA'

e. Vidal lá,

Vidal $\mathrm{la}^{\mathrm{H}}$

Vidal LA

'Vidal LA'

f. ne náa

$n \mathrm{e}^{\mathrm{LH}}$ naa

y $1 \mathrm{SG}$

'Y YO'

g. bide'du

bi-de' $=\mathrm{du}^{\mathrm{LH}}$

jmá cáguăma

$\mathrm{jma}^{\mathrm{H}}$ cagua $^{\mathrm{LH}} \mathrm{ma}$

COMPL-beber=1PL.EXCL mucho cerveza

'Bebimos mucha cerveza'

LA aparece en (24b) al final de un modificador adverbial (similar a los usos mencionados anteriormente en (17) y (18)). En (23d), el hablante usa el pronombre independiente de tercera persona seguido de LA para referirse a uno de sus interlocutores ( $\mathrm{y}$ al que señala simultáneamente usando su dedo). En la siguiente línea (23e), se refiere a otro referente de tercera persona (no a un participante en la conversación) usando su primer nombre seguido de LA. Él agrega un referente final, a sí mismo (23f), sin el uso de LA. Esos tres individuos componen un grupo, establecido en tres unidades entonacionales separadas, que funciona como el tópico del enunciado en (23g), representado por el clítico =DU (1PL.EXCL). De esta forma, el hablante utiliza la partícula LA para ayudar al oyente a identificar a los individuos en cuestión. Es decir, establece un contexto compartido, antes de la predicación en (23g) (cf. Lambrecht 1994, 184-191). 


\subsection{Resumen}

Es importante señalar que el análisis del habla espontánea y, específicamente, de la conversación, hace posible un análisis multifuncional de la partícula LA. A través de este análisis, vimos que las construcciones marcadas con LA pueden tener una función de scene-setting topic, es decir, de establecer el marco espacial o temporal dentro del cual se sostiene la predicación.

Además, las construcciones con LA forman parte de las presuposiciones compartidas entre los participantes de un acto de habla, las cuales establecen un marco dentro del cual proceder con el discurso, de la misma manera que una pregunta. De hecho, LA se utiliza en preguntas de sí/no para asegurar un punto de referencia común con el oyente. Como tal, LA se puede ver no solo como un recurso para marcar varios tipos de información topical, sino más generalmente como un recurso para organizar la conversación y la interacción.

\section{Resumen y conclusiones}

En este trabajo, se exploró el rango de tipos de construcciones de foco en los datos del ZAI. Se mostró, por un lado, que en la estructura informativa de ZAI las construcciones de foco oracional y de foco de predicado son consistentemente de verbo inicial. Por otro lado, se vio también que las construcciones de foco estrecho pueden ser construcciones de verbo inicial o tener constituyentes preverbales (dentro de la cláusula). Un resumen de estas observaciones se presenta en la Tabla 2:

Tabla 2: Las construcciones focales en ZAI.

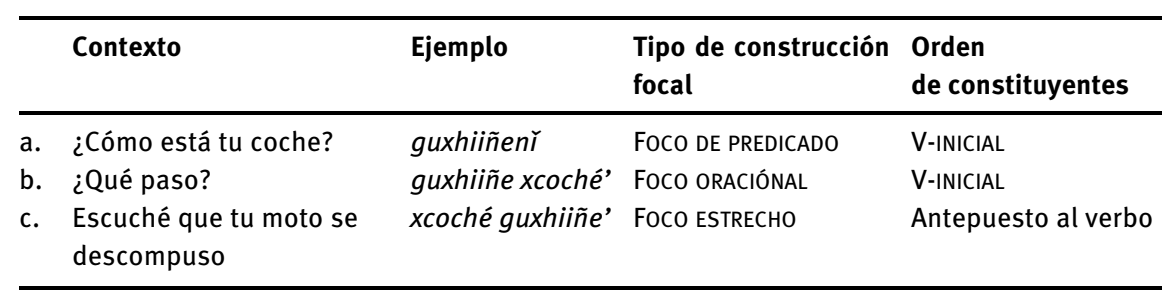

Se mostró, además, que no hay evidencia de que haya algún tono directamente asociado con el material focal. Sin embargo, los elementos pueden mostrar varias propiedades prosódicas: mayor duración, mayor registro de tono y mayor rango de tono relacionado con su posición dentro de una unidad entonacional 
dada. En particular, los elementos en foco, ya sean constituyentes nominales o verbales, tienden a ocurrir en posiciones prosódicamente más prominentes, es decir, al principio de la unidad entonacional. Asimismo, la prominencia prosódica de inicio de frase quizás sirva como motivación para que el dominio de foco se asocie principalmente con la posición inicial (ya sea el verbo en una construcción verbal inicial o un elemento preverbal de otro tipo).

Estas observaciones nos llevaron a examinar el lugar de ZAI dentro de la tipología de la estructura de foco propuesto por Van Valin (1999). En primer lugar, dado que los argumentos pueden ocurrir antes o después del verbo, se describió al ZAI como relativamente flexible sintácticamente. En segundo lugar, dado que los constituyentes en foco pueden aparecer de forma preverbal o posverbal (menos los sujetos transitivos en foco), se determinó que el potencial dominio de foco en ZAI también es relativamente flexible.

En construcciones de foco amplio (es decir, de foco oracional o de foco de predicado), el dominio de foco es posverbal. En construcciones de foco estrecho, hay una fuerte preferencia por que los constituyentes en foco aparezcan preverbalmente. Los sustantivos léxicos, ya sean preverbales o posverbales, generalmente son parte del dominio de foco, al igual que los pronombres preverbales independientes. En contraste, los enclíticos pronominales siempre son tópicos.

Sin embargo, se observó que la estructura de foco es más rígida que la sintaxis, ya que la estructura de foco puede motivar ciertas disposiciones sintácticas, mientras que el reverso nunca se cumple. Dicho de otra forma, la estructura sintáctica no parece motivar cambios en el dominio de foco.

Partiendo de este análisis de la estructura focal del ZAI, en la Sección 3 se amplió la discusión para detenerse en una estrategia conversacional utilizada por los hablantes de ZAI, que involucra el uso sucesivo del foco de predicado y del foco estrecho para lograr objetivos conversacionales específicos. El uso de la construcción de foco de predicado seguido inmediatamente por una construcción de foco estrecho se analizó como una estructura quiástica que explota el paralelismo de las estructuras sintácticas. Al usar esta estructura quiástica, las dos unidades entonacionales se unen para ser interpretadas juntas, y el hablante amplía su turno de habla por una unidad entonacional adicional, con la segunda parte, la construcción del foco estrecho, marcando el final del turno del hablante, que cede la palabra.

En la Sección 4, se mostró que el uso del morfema LA debe entenderse como un recurso no solo para la marcación de elementos que "fijan la escena" (scene-setting topics) sino, más generalmente, como un elemento cuya función es organizar la conversación de modo que esa organización sea reconocida por los participantes del acto de habla. Se demostró que un análisis completo de LA depende del análisis del habla espontánea $\mathrm{y}$, especialmente, de la 
conversación. Podría ser útil en el futuro investigar el uso de LA como un recurso en la construcción conjunta de la comunicación, en la toma de turnos y, de manera más general, como una ventana hacia la forma en la que los hablantes del ZAI se orientan a la conversación y a la interacción.

Debido a que los oyentes en diferentes comunidades de habla pueden orientarse de diferentes maneras, se propone la siguiente pregunta: ¿Cómo se puede vincular el uso de la partícula LA con las estrategias y normas conversacionales locales? Desde esta perspectiva, probablemente una caracterización de LA, así como una caracterización más general de la estructura focal del ZAI en términos de nociones como tópico y foco sea insuficiente (cf. Matić y Wedgwood 2013). En cambio, es probable que se llegue a entender mejor los usos de LA y los usos de la estructura focal a través de un análisis de la interacción; es decir, a través de un análisis de los tipos de interacciones que los participantes están teniendo en la conversación y por qué.

\section{Agradecimientos}

Agradezco profundamente a la comunidad de Juchitán y, en particular, a Tomás Villalobos Aquino y familia por compartir conmigo su conocimiento, su trabajo, y su entusiasmo por el diidxazá. Agradezco también al programa de becas de Endangered Languages Development Program (ELDP) y al programa Documenting Endangered Languages de la National Science Foundation (NSF-DEL) por su apoyo durante el trabajo de campo y la recolección de datos.

\section{Referencias}

Alfabeto popular para la escritura del zapoteco del Istmo. 1956. México: Sociedad ProPlaneación Integral del Istmo.

Beam de Azcona, Rosemary. 2004. A Coatlán-Loxicha Zapotec Grammar. Tesis de doctorado. University of California, Berkeley.

Belloro, Valeria y Lilian Guerrero. 2012. La interfaz gramática-discurso en las construcciones de movimiento en yaqui. Lingüística 27. 197-220.

Broadwell, George A. 1999a. Focus alignment and optimal order in Zapotec. En Sabrina Billings, John Boyle y Aaron Griffith (eds.). Proceedings of the 35th Chicago Linguistics Society, 15-28. Chicago: CLS 35.

Broadwell, George A. 1999b. The interaction of focus and constituent order in San Dionicio Ocotepec Zapotec. En Miriam Butt y Tracy Holloway King (eds.). Proceedings of the LFG 99 conference. Manchester: University of Manchester. 
Broadwell, George A. 2002. Preverbal positions and phrase boundaries in Zapotec. Ponencia. Annual meeting of the Linguistic Society of America. San Francisco: Linguistic Society of America.

Bueno Holle, Juan José. 2016. Information structure in Isthmus Zapotec. Tesis doctoral. University of Chicago.

Bueno Holle, Juan José. 2019. Information structure in Isthmus Zapotec narrative and conversation. Berlin: Language Science Press.

Castillo Hernández, Carolina. 2014. Observaciones sobre la interfaz sintaxis-pragmática en narrativas de tres lenguas indígenas mexicanas. Signo y Seña 25. 35-58.

Enríquez Licón, Maritza. 2009. Clases verbales en zapoteco del Istmo. En Ausencia López Cruz y Michael Swanton (eds.). Memorias del Coloquio Internacional de Lingüística Mauricio Swadesh, 365-375. Oaxaca: Biblioteca Francisco de Burgoa.

Fox, James. 1977. Roman Jakobson and the comparative study of parallelism. En Daniel Armstrong (ed.). Roman Jakobson: Echoes of his scholarship, 59-90. London: Peter de Ridder Press.

Gibbs, William. 1977. Discourse elements in Sierra de Juárez Zapotec. Tesis de maestría. University of Texas.

Haiman, John. 1978. Conditionals are topics. Language 54. 564-589.

Jakobson, Roman. 1966. Grammatical parallelism and its Russian facet. Language 42. 399-429.

Kerslake, Celia. 1996. Future time reference in subordinate clauses in Turkish. En Kamile İmer y N. Engin Uzun (eds.). Proceedings of the VIIIth International Conference on Turkish Linguistics, 49-59. Ankara: Ankara University.

König, Ekkehard y Peter Siemund. 2007. Speech act distinctions in grammar. En Timothy Shopen (ed.). Language typology and syntactic description, 276-324. Cambridge: Cambridge University Press.

Konnerth, Linda. 2013. Additive particle and discourse contrast marker: Evidence from Karbi (Tibeto-Burman) =ta. Ponencia. Taller Information Structure in Spoken Language Corpora. Bielefeld: University of Bielefeld.

Lambrecht, Knud. 1994. Information structure and sentence form. Cambridge: Cambridge University Press.

Lee, Felicia. 1997. Focus and Judgment Type in San Lucas Quiaviní Zapotec. BLS 23. 234-244.

Lee, Felicia. 2000. VP remnant movement and VSO in Quiavini Zapotec. En Andrew Carnie y Eithne Guilfoyle (eds.). The syntax of verb-initial languages, 143-162. Oxford: Oxford University Press.

Lillehaugen, Brooke D. 2008. El morfema làa' y su uso en el zapoteco de Tlacolula de Matamoros. Ponencia. Encuentro Internacional de Lingüística en el Noreste. Hermosillo, Sonora: Universidad de Sonora.

Lillehaugen, Brooke D. 2016. The syntax of preverbal subjects in Colonial Valley Zapotec. Ponencia. VII Syntax of the World's Languages: International Conference and Workshop. Mexico: UNAM.

Marlett, Stephen A. y Velma Pickett. 1987. The syllable structure and aspect morphology of Isthmus Zapotec. International Journal of American Linguistics 53. 398-422.

Matić, Dejan y Daniel Wedgwood. 2013. The meanings of focus: The significance of an interpretation-based category in cross-linguistic analysis. Journal of Linguistics 49(1). 127-163.

Mithun, Marianne. 1992. Is basic word order universal. En Doris Payne (ed.). Pragmatics of word order flexibility, 15-61. Philadelphia: John Benjamins. 
Munro, Pamela y Felipe López. 1999. Di'csyonaary X: tèe’n Dii'zh Sah Sann Lu'uc: San Lucas Quiaviní Zapotec Dictionary. Los Angeles: UCLA Chicano Studies Research Center.

Munro, Pamela, Brooke D. Lillehaugen y Felipe López. 2008. Cali Chiu?: A Course in Valley Zapotec. Los Angeles: Lulu Publishing.

Öpengin, Ergin. 2013. Topicalisation in Central Kurdish: additive particle and other means. Ponencia. Taller Information Structure in Spoken Language Corpora. Bielefeld: University of Bielefeld

Ozerov, Pavel. 2015. Information structure without topic and focus: Differential object marking in Burmese. Studies in Language 39(2). 386-423.

Payne, Doris. 1990. The pragmatics of word order: typological dimensions of verb initial languages. Berlin/Boston: De Gruyter.

Pekarek Doehler, Simona. 2011. Emergent grammar for all practical purposes: the on-line formatting of left and right dislocations in French conversation. En Peter Auer y Stefan Pfänder (eds.). Constructions: Emerging and Emergent, 45-87. Berlin/Boston: Mouton de Gruyter.

Pérez Báez, Gabriela. 2015. Morphological valence-changing processes in Juchitán Zapotec. En Natalie Operstein y Aaron H. Sonnenschein (eds.). Valence Changes in Zapotec: Synchrony, diachrony, typology, 110-93. Amsterdam/Philadelphia: John Benjamins.

Piper, Michael J. 1995. The functions of lëë in Xanica Zapotec narrative discourse with some implications for comparative Zapotec. SIL Mexico Workpapers 11. 67-78.

Picket, Velma. 1979. Vocabulario zapoteco del Istmo. Ciudad de México: Instituto Lingüístico de Verano.

Pickett, Velma, Cheryl Black y Vicente Marcial Cerqueda. 1998. Gramática popular del zapoteco del Istmo. Oaxaca: Centro de Investigación y Desarrollo Binnizá/Instituto Lingüístico de Verano.

Riggs, David B. 1987. Paragraph analysis for Amatlán Zapotec. SIL Mexico Workpapers 9. 1-11.

Riggs, David B. y Stephen A. Marlett. 2010. The le'e focus phrase: structural aspects. En Cheryl Black, H. Andrew Black y Stephen A. Marlett (eds.). The Zapotec grammar files. Instituto Lingüístico de Verano. En línea https://www.sil.org/resources/archives/60868 [23/04/2019].

Sacks, Harvey, Emmanuel A. Schegloff y Gail Jefferson. 1974. A simplest systematics for the organization of turn-taking for conversation. Language 50. 696-735.

Sicoli, Mark A., 2007. Tono: A linguistic ethnography of tone and voice in a Zapotec region (Mexico). Tesis de doctorado. University of Michigan.

Silverstein, Michael. 1984. On the pragmatic "poetry" of prose: Parallelism, repetition, and cohesive structure in the time course of dyadic conversation. En Deborah Schiffrin (ed.). Meaning, form, and use in context: Linguistic applications, 181-199. Washington, DC: Georgetown University.

Sonnenschein, Aaron H. 2005. A descriptive grammar of San Bartolomé Zoogocho Zapotec. München: LINCOM.

Thompson, Sandra A., Robert E. Longacre y Shin Ja J. Hwang. 2007. Adverbial clauses. En Timpothy Shopen (ed.). Language typology and syntactic description, 237-300. Cambridge: Cambridge University Press.

Van Valin, Robert D. Jr. 1999. A typology of the interaction of focus structure and syntax. En Ekaterina Raxilina y Yakov Testelec (eds.). Typology and the Theory of Language: From Description to Explanation, 511-524. Moscow: Languages of Russian Culture.

Ward, Michael. 1987. A focus particle for Quioquitani Zapotec. SIL Mexico Workpapers 9. 26-32. 
